\title{
Nivel de satisfacción de estudiantes en el diseño e implementación del laboratorio de simulación virtual en la Sección de Farmacología de la Facultad de Medicina de la UNMSM
}

\author{
Maritza Dorila Placencia Medina¹, Carlos García Bustamante², Hubert James Mendoza Rojas², Leonor María \\ Tenorio Salas 3 , Javier Silva Valencia ${ }^{4}$, Jorge Carreño Escobedo ${ }^{5}$
}

\section{RESUMEN}

Objetivo: Diseñar y construir un modelo pedagógico de uso del Laboratorio de Simulación Virtual en la Sección de Farmacología y conocer el nivel de satisfacción de los estudiantes.

Material y Métodos: Estudio descriptivo, cualitativo de tipo investigación-acción. Se planificó el diseño e implementación de un laboratorio de simulación virtual, la intervención de mejora y perfección del material didáctico para el desarrollo de prácticas en la sección de Farmacología de la Facultad de Medicina de la UNMSM, utilizando los software Microlab® y Rat CV. Se realizó la técnica de la entrevista, como instrumento se utilizó una encuesta de satisfacción de usuario del laboratorio virtual, utilizando una escala de Likert del mínimo de satisfacción iniciando en 1 al máximo de 10. Tamaño de muestra de 26 alumnos.

Resultados: Se implementó un nuevo proceso de enseñanza - aprendizaje de Farmacología Experimental mediante el uso del Laboratorio de simulación virtual. La mayoría de los alumnos encuestados sobre la implementación del laboratorio de aula virtual, mostró un nivel de satisfacción de 9-10/10 en la escala de Likert.

Conclusión: El laboratorio de simulación virtual de la Sección de Farmacología implementado estimula el aprendizaje eficiente de los actores de este proceso, con un buen nivel de satisfacción de los estudiantes. (Horiz Med 2015; 15(3): 51-56)

Palabras clave: Simulación virtual, aprendizaje aulas virtuales, investigación-acción. (Fuente: DeCS BIREME).

Satisfaction level of students in the design and Implementation of a virtual simulation laboratory in the Department of Pharmacology, Faculty of Medicine at UNMSM

\section{ABSTRACT}

Objective: To design and build an educational model using a Virtual Simulation Laboratory Section of Pharmacology and to know the level of student satisfaction.

Material and Methods: Descriptive, qualitative study of research-action type. The design and implementation of a virtual simulation laboratory, the intervention of improvement and perfection of teaching materials for the development of the practical section of Pharmacology, Faculty of Medicine at San Marcos was planned, using the software Microlab ${ }^{\circ}$ and CV Rat. A survey of user satisfaction in virtual laboratory technique was conducted, using the interview as an instrument, and a Likert scale of minimum satisfaction starting in 1 to maximum of 10 . Sample size of 26 students.

Results: A new process of Experimental Pharmacology Laboratory teaching - learning was implemented, using virtual simulation. Most students in the virtual classroom lab have a satisfaction level 9-10 / 10 on the Likert scale.

Conclusion: The implemented virtual simulation laboratory in the Section of Pharmacology, efficiently stimulates the learning of the actors of this process, with a good level of student satisfaction. (Horiz Med 2015; 15(3): 51-56)

Key words: virtual simulation, virtual classroom learning, action-research. (Source: MeSH NLM).

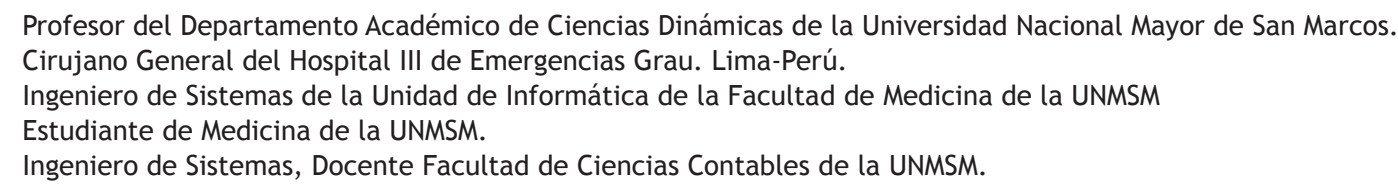




\section{INTRODUCCIÓN}

Entre los años 2011-2012, se introdujo en el curso de Farmacología de la EAP de Medicina de la UNMSM tres prácticas de simulación virtual con el Software Microlab $\AA$, software donado en el 2010 por el Dr. Hendrik van Wilgenburg.

La necesidad de desarrollar un laboratorio de simulación virtual en Farmacología se basó en tres motivos fundamentales:

1. El alto costo de los bioterios con las medidas de bioseguridad y con la genotipación de las cepas de animales para la docencia e investigación.

2. El tiempo que demora los procesos de implementación y certificación de gestión de calidad en la administración de los bioterios.

3. Evitar los riesgos a los que pueden estar expuestos los estudiantes en un laboratorio real, sin protocolos de bioseguridad y sin el equipamiento para la experimentación en Farmacología.

El uso de la simulación virtual permite al estudiante un aprendizaje interactivo con creatividad y claridad en la fundamentación teórica de la farmacocinética y farmacodinamia de las drogas.

La enseñanza de Farmacología es uno de los retos más importantes para las universidades públicas en todo el mundo $(1,2,3)$. Actualmente, los métodos alternativos y la simulación virtual son reconocidos como parte vital de la metodología de enseñanza ya que aseguran el aprendizaje en el laboratorio, reducen costos y respetan los principios éticos para con los animales $(4,5)$.

Aunque se ha venido creyendo que el único método válido para la enseñanza es el uso de animales y que el empleo de programas alternativos disminuye la calidad de la docencia, diversos estudios comparativos han señalado que el uso de estos modelos virtuales no disminuyen el aprendizaje, sino que incluso pueden llegar a aumentarlo $(6,7$, $8)$.

Las experiencias en otros campos del conocimiento como la de Márquez (9), Meza (10) y Riofrío y col. (11), nos muestran el uso del que otras ciencias le están dando a las herramientas virtuales, como lo es el caso del Laboratorio Virtual, medio por el cual han podido potenciar, enriquecer y mejorar la didáctica de la enseñanza y del aprendizaje del estudiante.

Meza (10) lo incorporó y lo implementó en los trabajos prácticos de laboratorio de física universitaria básica.

El poder trabajar con modelos o simulaciones donde el alumno toma parte activa en una serie de decisiones en función de determinados sucesos, o cambiar variables con vistas a la comprobación de hipótesis previas, son formas de trabajo intelectual enriquecidas por el aporte de la computadora.

Riofrío (11) describe el diseño y la implementación de un laboratorio virtual de biotecnología, en el que uno o más estudiantes pueden realizar una práctica de ingeniería genética que consiste en modificar genéticamente un árbol (chopo) para dotarlo de una mayor resistencia a enfermedades, especialmente las producidas por hongos.

La integración del e-learning con los Mundos Virtuales Moodle $(9,10)$ nace como evolución de la tecnología web y la proliferación del uso de Internet.

Moodle fue creada por Martin Dougiamas y lanzada en 2002, es una aplicación web gratuita de tipo Ambiente Educativo virtual, que ayuda a crear y administrar sitios web dinámicos de aprendizaje colaborativo donde los profesores pueden crear sitios para sus asignaturas, y así poder publicar material y noticias de la asignatura, o crear tareas y exámenes que los alumnos puedan realizar desde su casa.

Según Kumar y col. (12), la arquitectura de Moodle está compuesta por módulos independientes que se pueden reunir en seis grupos diferentes de acuerdo a su propósito: módulos de comunicación, módulos de productividad, módulos de participación de los estudiantes, módulos de administración, módulos de entrega del curso y módulos de diseño de currículo.

Ellos realizaron una comparativa con otros sistemas de e-learning en cuanto a la arquitectura y características técnicas, y llegaron a la conclusión de que el mejor es Moodle, a pesar de sus defectos de seguridad. 
El presente estudio tuvo como objetivo diseñar y construir un modelo pedagógico de uso del Laboratorio de Simulación Virtual en la Sección de Farmacología y conocer el nivel de satisfacción de los estudiantes.

\section{MATERIAL Y MÉTODOS}

Es una investigación descriptiva, cualitativa de tipo investigación-acción.

La arquitectura de la plataforma virtual en la web de San Fernando UNMSM en línea de la Sección de Farmacología DACD, se desarrolló mediante el uso de la plataforma educativa virtual Open Source denominada Moodle.

Se realizó la implementación de 06 prácticas con el software de Microlab ${ }^{\circ}$ (Figura 1, 2 y 3).

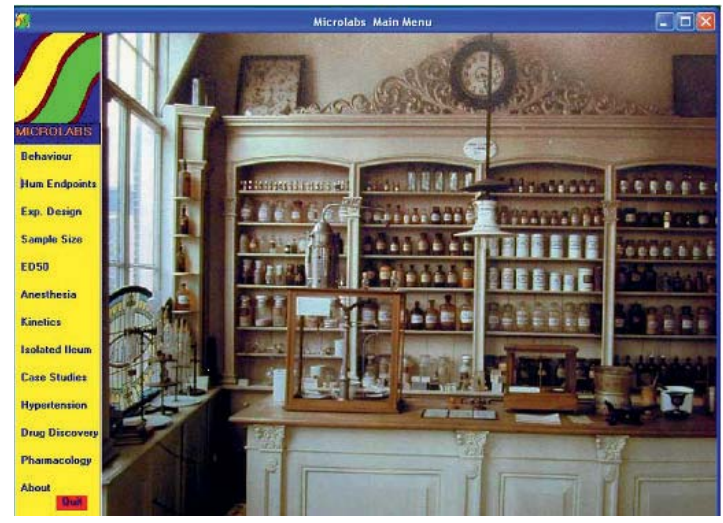

Figura 1. Ventana de la Portada de software Microlab®

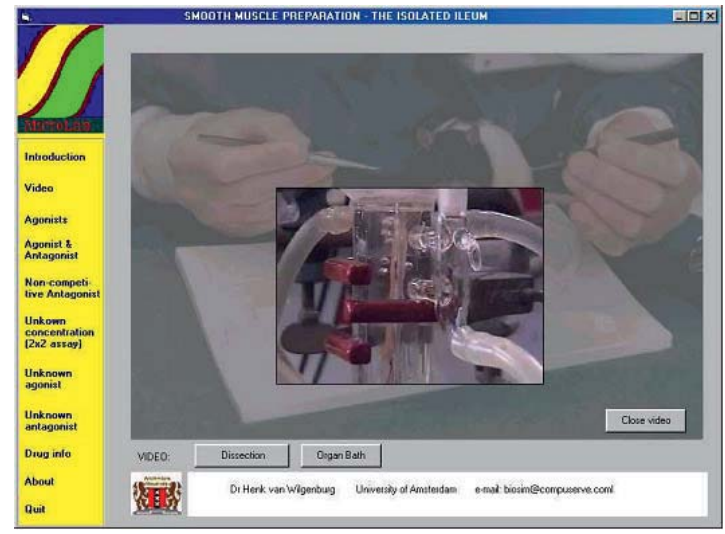

Figura 2. Administración de drogas en el baño de órganos.

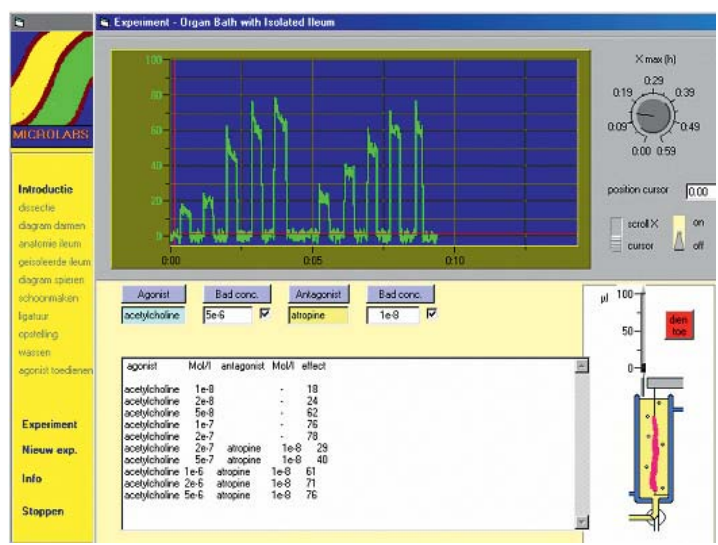

Figura 3. Resultados de la práctica de Acetilcolina.

Finalmente se realizó una encuesta de satisfacción de los laboratorios virtuales.

Esto formó parte de la investigación-acción, utilizando una encuesta anónima y de carácter social, para la mejora continua del curso.

Se tomó una muestra no aleatoria de 26 estudiantes del curso de Toxicología del 2013 de la EAP de Tecnología Médica de la UNMSM.

Se utilizó la técnica de la entrevista y como instrumento una encuesta de satisfacción de usuario, utilizando una escala de Likert del mínimo de satisfacción iniciando en 1 al máximo de 10.

Se consideraron en la evaluación integral de toda la actividad del Laboratorio-Taller las dimensiones de las 4 áreas importantes:

1. Evaluación de la organización del curso

2. Evaluación del Aula virtual

3. Evaluación del Taller en el auto-aprendizaje

4. Evaluación del / de la Facilitador (a) 


\section{RESULTADOS}

Se implementó un nuevo proceso de enseñanza - aprendizaje de Farmacología Experimental mediante el uso del Laboratorio de simulación virtual.

Los 18 docentes de la sección de Farmacología fueron capacitados en el manejo del software Microlab ${ }^{\circledR}$, y el uso de TICs en la docencia.

Los resultados obtenidos de la encuesta de satisfacción de los estudiantes fueron:

En la evaluación de la organización del aula virtual, 10 estudiantes mostraron satisfacción en la organización de 9/10; Ocho presentaron una satisfacción de 9/10 en la evaluación del aula virtual. Nueve, tuvieron una satisfacción 9/10 con respecto al autoaprendizaje utilizando el taller (Figura 4).

La evaluación del facilitador, mostró que la mayoría de estudiantes encuentra una satisfacción de 10/10. La puntuación más alta.

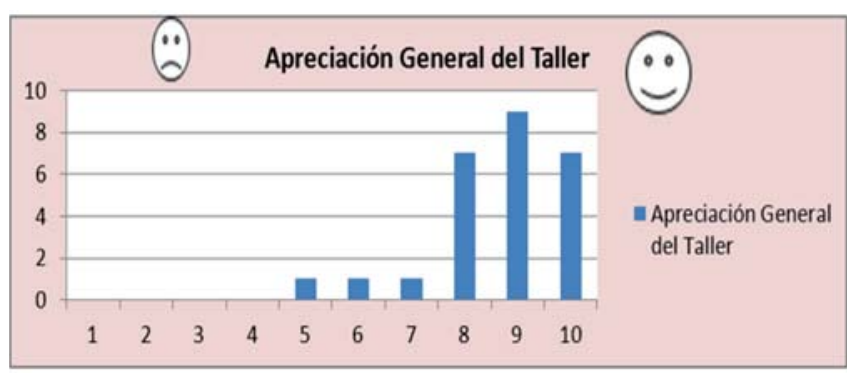

Figura 4. Evaluación del Taller

De esta manera, existió una satisfacción con el taller de aprendizaje de Toxicología usando Microlabs ${ }^{\circledR}$, por la facilidad de su manejo y la claridad de los conceptos básicos de farmacocinética y farmacodinamia de los grupos farmacológicos experimentados, la programación interactiva de su contenido a través de videos, dibujos esquemáticosy sistemas de registros de la actividad farmacológica, su uso en cualquier lugar y en cualquier horario, motivando el aprendizaje de la farmacología e investigación.

\section{DISCUSIÓN}

En la UNMSM, se ofrecen carreras en las áreas de las ciencias de la salud, y muchas de las materias contempladas dentro del plan curricular cuentan con una parte teórica y otra práctica. La modalidad práctica normalmente consiste en la realización de un laboratorio.

El "paquete instructivo" de las materias con laboratorio incluye un manual de prácticas de laboratorio, el estudiante encuentra en él todas las prácticas que se desarrollarán en el curso de Farmacología. Los laboratorios son estructurados de manera que el educando estudie con anticipación cada una de las prácticas correspondientes, para que las realice en el laboratorio y después entregue un informe escrito, según el formato establecido por cada asignatura (normalmente este incluye resultados, discusión, conclusiones y respuestas a los ejercicios o cuestionario).

La realización del laboratorio consiste, por ejemplo, en que el estudiante observe el comportamiento de una droga al ser administrada a un animal de experimentación (rata o ratón albino); siendo " $X$ "; la droga de uso para "Y" (ratón o rata); ésta preparación del material, la realiza el docente con el técnico en farmacología; el estudiante observa lo que sucede.

La manipulación y preparación de los animales de experimentación in situ (modelo de órganos aislados), los reactivos, y drogas utilizadas que serán observadas por el estudiante, son preparados por los docentes o los técnicos de farmacología.

En la UNMSM no existen las condiciones para que el estudiante prepare los reactivos, utilice los animales del bioterio certificado, y prepare los tejidos u órganos aislados para que pueda observar, analizar y discutir lo observado.

Cada práctica de laboratorio se lleva a cabo en una fecha prefijada, con horario fijo y en los Laboratorios Experimentales, previamente establecidos por la coordinación de programas de 
laboratorios experimentales de las asignaturas en la Sección de Farmacología.

Estas actividades de laboratorio son consideradas como un elemento indispensable del quehacer académico en las carreras de las ciencias de la salud, razón por la cual cuentan con un valor porcentual dentro de la evaluación del curso.

Con estas experiencias se mantiene un contacto "cara a cara" entre los estudiantes y el profesor, y en algunas oportunidades con el técnico, pero muchas veces ello es impersonal debido a que en una misma sesión de laboratorio se realizan hasta dos prácticas de experimentos, lo que reduce el tiempo para las discusiones y tutorías presenciales.

La diferencia del laboratorio virtual con un laboratorio convencional de Farmacología está en que el estudiante no debe asistir a un espacio físico para realizar las prácticas virtuales, ni a un horario establecido; por el contrario, podrá realizar cada una de las actividades desde cualquier computadora personal: la suya, la de un amigo, o inclusive la del aula de telemática en San Fernando porque tiene instalado el programa de Microlab ${ }^{\circledR}$ y en el horario que más le convenga, para aprender Farmacología respetando la vida de los animales de experimentación.

Este laboratorio virtual usando Microlab ${ }^{\circledR}$ tiene una estructura electrónica más elaborada, pero a través de las guías y tutoriales informatizados siempre mantiene el mismo formato que los otros laboratorios: objetivos, explicación, actividades, descripción de cada uno de los experimentos que se van a analizar en el laboratorio y preguntas de autoevaluación.

En un laboratorio experimental de farmacología, para que el estudiante comprenda y analice, la interacción entre una droga y un ser vivo (ratón, conejo o rata), se le entrega una guía previamente elaborada, los reactivos de la práctica realizado por el docente responsable, la especie a ser observada trabajada por el técnico de laboratorio y luego se realiza la práctica.

Un laboratorio de simulación virtual es una práctica que se observa en la pantalla del computador y que solo existe como información lo que se observa y se analiza mediante una base teórica previa.

El Microlab® programa el laboratorio virtual diseñado por Henk van Wilgenburg (8), se instala en la computadora, y funciona sin internet; de manera que no se mantiene una comunicación real con el profesor fuera del aula, pues el laboratorio ha sido estructurado de tal forma que le permita al estudiante realizarlo desde cualquier computadora personal; solo accedemos a la red para el ingreso al aula virtual tal como sucede con otras experiencias en clases virtuales de universidades a distancia (13, 14).

En esta investigación, se ha trabajado con el software Microlab $₫$, y se han desarrollado 04 tutoriales y 09 guías de prácticas virtuales para docentes y estudiantes, las cuales al ser practicadas pueden dominar del programa del laboratorio de Microlab $®$ y de otros software libres que motivan al auto-aprendizaje de Farmacología y Toxicología.

Contamos en nuestra plataforma web con el aula virtual Moodle 2.5.2, donde los estudiantes tienen a disposición el repositorio de la documentación de la asignatura, y envían sus productos de aprendizaj e para su evaluación en línea. De esta manera, estamos ingresando a la modernidad en el uso de las TICs como herramientas para el aprendizaje al igual que otras universidades innovadoras en nuestro medio nacional e internacional.

\section{Fuentes de financiamiento}

Autofinanciado por los autores.

\section{Conflictos de interés}

Los autores declaran no tener conflictos de interés. 


\section{REFERENCIAS BIBLIOGRÁFICAS}

1. Rodríguez-Carranza R, Vidrio H, Campos-Sepúlveda E. La enseñanza de la farmacología en las escuelas de medicina. Situación actual y perspectivas. Gac Méd Méx 2008; 144(6):463-472.

2. O'Shaughnessy L, Haq I, Maxwell SRJ, Llewelyn MJ. Teaching of clinical pharmacology and therapeutics in UK medical schools: current status in 2009. Br J Clin Pharmacol 2010; 70:143-8.

3. Maxwell S, Walley T. Teaching safe and effective prescribing in UK medical schools: a core curriculum for tomorrow's doctors. Br J Clin Pharmacol 2003; 55:496-503. doi: 10.1046/j.13652125.2003.01878.x

4. Sefton A. International workshop: modern approaches to teaching and learning Physiology. Adv Physiol Educ 2001;25:64-71

5. Buchanan et al. Guidelines for the treatment of animals in behavioural research and teaching. Anim Behav 2012; 83:301-309.

6. Cheesman M, Chen S, Manchadi ML, Jacob T, Minchin R, Tregloan P. Implementation of a virtual laboratory practical class (VLPC) module in pharmacology education. Pharmacognosy communications 2014;4(1):2-10

7. Ezeala C, Ram A, Vulakouvaki. Learning gain of pharmacy students after introducing guided inquiry learning with computer simulation in a pharmacology class in Fiji. J Educ Eval Health Prof 2013;10:1-4

8. Corvetto $M$ y col. Simulación en educación médica: una sinópsis. Rev Med Chile 2013; 141:70-79.

9. Márquez D, Cárdenas O. Implementación de un Laboratorio Virtual para la enseñanza de Controladores PID. Información Tecnológica. 2008;19(3):75-78 doi:10.1612/inf.tecnol.3884it.07

10. Meza S, Aguirre S, Lucero L, Sampallo G, Concari S. Propuesta de Implementación de Laboratorio Virtual en Física. Memorias Comunicaciones Científicas y Tecnológicas. Corrientes, Argentina: Facultad de Ciencias Exactas y Naturales y Agrimensura. Universidad Nacional del Nordeste; 1999.

11. Riofrío Luzcando, D. Diseño e Implementación de un Laboratorio Virtual de Biotecnología. (Tesis Master). España. Archivo Digital UPM. Universidad Politécnica de Madrid; 2012.

12. Kumar, S, Gankotiya, AK, \& Dutta, K. A comparative study of moodle with other e learning systems. 3rd International Conference on Electronics Computer Technology (ICECT). Kanyamukari 2011, p. 414-418. doi:10.1109/ICECTECH.2011.5942032

13. Álvarez de Sotomayor M, Pérez-Guerrero C, Herrera MD. Dos diferentes niveles de empleo de docencia virtual en el departamento de farmacología de la Universidad de Sevilla: E-learning y B-learning. Edusfarm, revista d'educació superior en Farmàcia 2008;3:1-11

14. Encinas M, Ros-Rodriguez JM, Gilabert JA. Recursos y actividades en el marco de un seminario de b-learning de Farmacología dentro del campus virtual UCM. Relada 2010;4(4):257-265.

\section{Correspondencia:}

\section{Maritza Dorila Placencia Medina}

Dirección: Av. Del Pacifico 135 Int. E2 Dpto. 802.

San Miguel. Lima, Perú.

Celular: 999218669

Correo: maritza.placencia@gmail.com

mplacenciam@unmsm.edu.pe 